\title{
Modelling and Simulation Approaches for Lightweight Components and Systems in Multi- Material Design Employing Scale-Spanning Methods
}

ISSN: 2576-8840

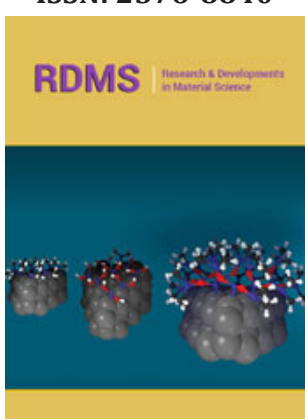

*Corresponding author: Andreas Hornig, Technische Universität Dresden, Institute of Lightweight Engineering and Polymer Technology, Germany

Submission: 齿 April 10, 2019

Published: 眥 April 17, 2019

Volume 10 - Issue 4

How to cite this article: A. Hornig, D. Weck, B. Grüber, M. Gude. Modelling and Simulation Approaches for Lightweight Components and Systems in MultiMaterial Design Employing Scale-Spanning Methods. Res Dev Material Sci. 10(4). RDMS.000743.2019.

DOI: $10.31031 /$ RDMS.2019.10.000743

Copyright@ Andreas Hornig, This article is distributed under the terms of the Creative Commons Attribution 4.0 International License, which permits unrestricted use and redistribution provided that the original author and source are credited.

\section{Andreas Hornig*, Daniel Weck, Bernd Grüber and Maik Gude}

Technische Universität Dresden, Institute of Lightweight Engineering and Polymer Technology (ILK), Germany

\section{Introduction}

The development of modern lightweight structures and systems requires both a better exploitation of the full potential of the material and simultaneously an increased cost efficiency. Already today, computer-based design and calculation methods deliver a crucial contribution to reach this aim. In the future, they will even get stronger into the focus of the whole development process. Therefore, the synergetic combination of different simulation methods along the entire value-added chain is a key to efficient lightweight structures.

\section{Dimensioning of resource-efficient lightweight components in multi-material design}

The design process for lightweight components and systems in multi-material design differs fundamentally from the design process of structures made of conventional isotropic materials. In contrast to established approaches, designing with fiber-reinforced composites is governed by a complex interaction between engineering, fabrication and verification. Currently, such a design process is still characterized by a trial-and-error approach. Compared to classical materials, it becomes apparent that higher levels of detail need to be considered when dealing with composite materials.

The fibre-reinforced material itself exhibits a hierarchical structure. On the micro scale the material is characterized by the properties of the reinforcing filaments and of the matrix material. Subsequently, the roving and the textile reinforcement architecture define the properties of a single reinforced layer on the meso scale. Finally, combination and stacking of different layers result in the material characteristics on the macro scale and beyond on the structure level. Furthermore, this hierarchical view can be expanded by considering complex systems arising from joining components and by taking the mutual interaction of different structures into account. Additionally, for fibre- and textile-reinforced components and systems, the resulting properties are significantly controlled by the fabrication process and can be influenced purposefully hereby. Therefore, numerical modelling of the processing of $c$ materials and structures is an integral part of the scale spanning design process. Hence, already in an early development stage alternate variants of process management can be compared and evaluated with regard to process-efficiency and achievable material and structural properties.

For developing efficient lightweight-structures in multi-material design, joining and bonding zones are of particular importance. Therefore, in an increasing number of cases the necessity arises to incorporate such areas in detail into the simulation models for evaluating the global system behaviour realistically.

The rapidly increasing computing power of modern computer systems is a substantial basis for achieving the capability to synergistically combine and apply the appropriate 
analysis, modelling, discretisation and calculation methods across the different scales of fibre - reinforced materials and systems step by step. Therefore, in the near future it will be possible to take into account the complex structure-property relationship (Figure 1) along the whole process chain.

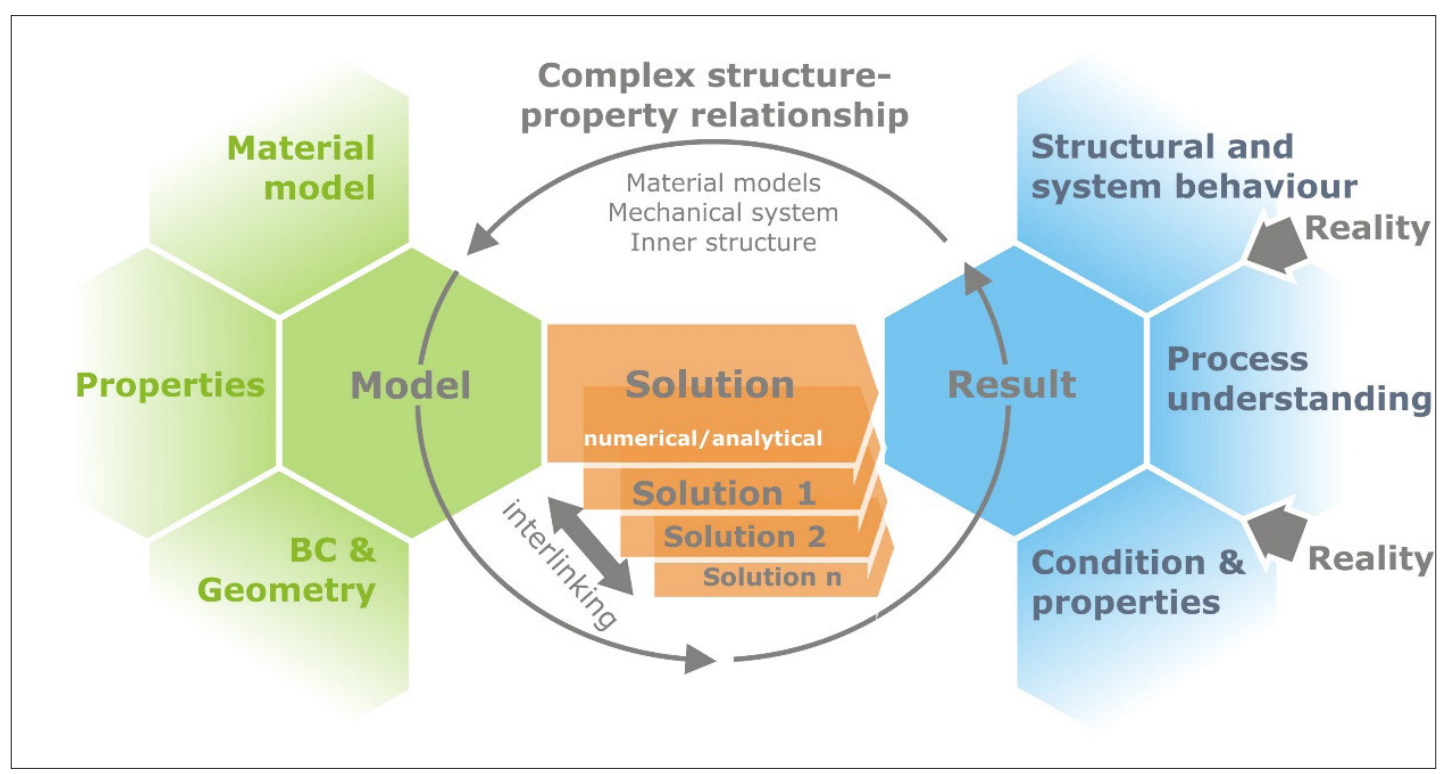

Figure 1: Complex structure-property relationship to be taken into account for simulation-based design of efficient lightweight structures in multi-material design.

\section{Material modelling - Scale-spanning methods for exploiting the material potential}

Choice of material is essential for structural design tasks. Based on mathematical approaches, a scientifically sound description of the complex composite material behaviour enables an accurate modelling of occurring physical phenomena. However, a closed solution of the resulting analytical framework is not always possible.
To overcome such obstacles, numerical material modelling within multiscale approaches is typically employed (Figure 2). This allows both an in-depth understanding of the material behaviour and the identification of relevant phenomena at the respective scales. The objective of a robust numerical material modelling route is to purposefully utilize the appropriate scale to describe the governing phenomena with adequate accuracy.
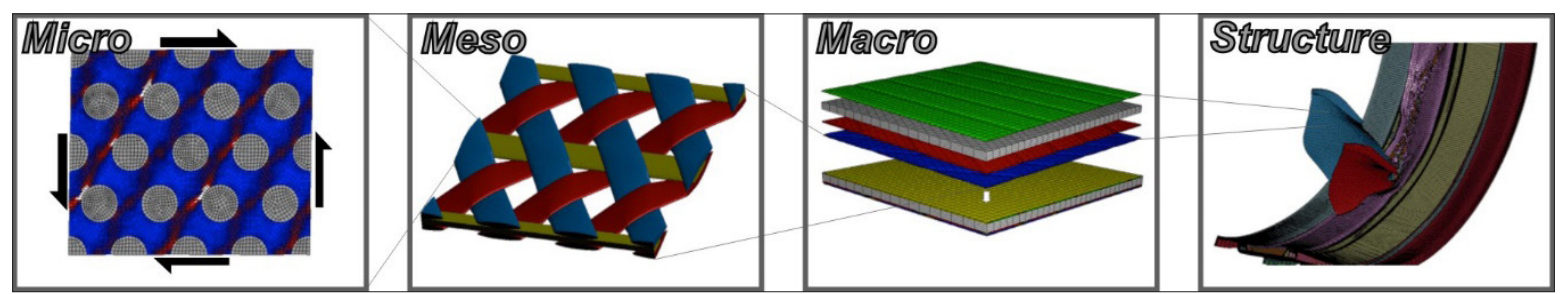

Figure 2: Scale-spanning modelling within FEM-a formula for success for reliable material modelling.

The basic constituents fibre (or filament) and matrix as well as their interactions in a matrix-embedded roving are numerically considered at the micro scale should be considered. The resulting detailed understanding enables meso-scale considerations on single-ply level to identify the influence of different textile architectures. Subsequently, mechanical properties derived for the single ply are adaptively homogenised within a multilayer composite framework on the macro scale. On the structural level, numerical analyses of composite components and (sub-) systems are performed using the multi-layer properties.
The basic idea of the multi-scale material modelling approach is to transfer relevant information from the neighbouring lower level scale into the working scale using homogenisation techniques. This enables for example the usage of filament-matrix interaction results for modelling a homogenised roving material to be used for the modelling of the textile reinforcement structure. In a next step on the macro scale, the ply interactions between the single plies are considered which can be subsequently used to investigate the delamination behaviour for instance. 


\section{Conclusion}

Efficient lightweight structures using up-to date multi-material design methods are getting more and more in the focus of $R \& D$ due to the permanent increasing demand on reduction of climatedamaging emissions and retrenchment of fossil energy carriers in many industrial sectors. For such lightweight structures, the design process is strongly influenced by a complex interaction of material, fabrication, joining and system behaviour. Up to now, the high material-specific potential is not fully exploited. Hence, the development of coupled material-, process- and applicationadapted simulation methodologies are expedited to fully utilize this potential. Striving for reliability, robustness, efficiency and sustainability of both the design process and the lightweight structure, tailored simulation strategies need to be developed combining in-house simulation tools with commercially available ones.

\section{Acknowledgment}

The "Saxon Alliance for Material- and Resource-Efficient Technologies" (AMARETO) is funded by the European Union (European Regional Development Fund) and the Free State of Saxony. 